\title{
The effects of liraglutide on liver enzymes and metabolic factors in patients with nonalcoholic steatohepatitis: a meta-analysis of randomized controlled trials
} \author{
Ans Ahmad Jajja ${ }^{3}$, Ahmad Khan ${ }^{7}$, James H. Tabibian ${ }^{8}$ \\ ${ }^{1}$ Loyola University Medical Center, Maywood, IL, USA \\ ${ }^{2}$ Harvard Medical School, Boston, MA, USA \\ ${ }^{3}$ Allama Iqbal Medical College, Lahore, Pakistan \\ ${ }^{4}$ Oklahoma University Medical Center, Oklahoma City, OK, USA \\ ${ }^{5}$ Hackensack University Palisades Medical Center, North Bergen, NJ, USA \\ ${ }^{6}$ Airedale General Hospital, Keighley, West Yorkshire, UK \\ ${ }^{7}$ Case Western Reserve University, Cleveland, OH, USA \\ ${ }^{8}$ Olive View-UCLA Medical Center, Sylmar, CA, USA
}

Adnan Malik ${ }^{1}$, Waseem Amjad², Faisal Inayat ${ }^{3}$, Mahum Nadeem $^{4}$, Simcha Weissman ${ }^{5}$, Muhammad Imran Malik ${ }^{6}$,

Gastroenterology Rev 2023; 18 (1): 100-109

DOI: https://doi.org/10.5114/pg.2022.112775

Key words: liraglutide, nonalcoholic steatohepatitis, liver enzymes, metabolic factors.

Address for correspondence: Adnan Malik MD, MPH, MBA, Loyola University Medical Center, 2160 S 1st Ave, Maywood, IL 60153, USA, phone: +1 (404) 786-8961, fax: +1 (888) 584-7888, e-mail: adnanmalik892@hotmail.com

\begin{abstract}
Introduction: Nonalcoholic steatohepatitis (NASH) is the most common cause of chronic liver disease, but no drug therapies have been approved to date. While glucagon-like peptide-1 (GLP-1) analogues may help in the management, the existing evidence remains conflicting.

Aim: This meta-analysis aims to elucidate the efficacy of liraglutide in patients with NASH.

Material and methods: We searched 4 databases for randomized controlled trials assessing the efficacy of liraglutide in patients with NASH. We analysed continuous outcomes using the mean difference (MD) and relative $95 \%$ confidence interval $(\mathrm{Cl})$, while dichotomous outcomes were analysed using the risk ratio (RR) and relative $95 \% \mathrm{Cl}$. Primary endpoints included alanine aminotransferase (ALT) (IU/I), aspartate aminotransferase (AST) (IU/l), alkaline phosphatase (ALP) (IU/I), and $\gamma$-glutamyl transferase (GGT) (IU/I). Secondary outcomes were body mass index (BMI) $\left(\mathrm{kg} / \mathrm{m}^{2}\right)$, waist circumference $(\mathrm{cm})$, total cholesterol (TC) $(\mathrm{mmol} / \mathrm{l})$, triglyceride $(\mathrm{TG})(\mathrm{mmoll})$, high-density lipoprotein $(\mathrm{HDL})(\mathrm{mmol} / \mathrm{l})$, low-density lipoprotein $(\mathrm{LDL})(\mathrm{mmol} / \mathrm{l})$, and glycated hemoglobin $\left(\mathrm{HbA}_{1 \mathrm{c}}\right)(\%)$.

Results: A total of 5 clinical trials were included. The analysis showed that liraglutide is effective in increasing HDL (MD = $+0.10(-0.18,-0.02), p=0.02)$ and reducing LDL levels in blood $(M D=-0.29(-0.56,-0.02), p=0.04)$. No significant difference was noted in levels of ALT $(M D=2.66(-1.56,6.87), p=0.22)$, AST $(M D=-1.99(-5.70,1.72), p=0.29)$, GGT $(M D=5.02(-0.86$, $10.90), p=0.09)$, ALP (MD $=-5.16(-11.90,1.59), p=0.13)$, TC $(\mathrm{MD}=-0.31(-0.65,0.03), p=0.07)$, or TG $(\mathrm{MD}=-0.14(-0.53$, $0.25), p=0.48)$. The $\mathrm{HbA}_{1 \mathrm{c}}(\%)$ level was found to be significantly reduced in the liraglutide arm $(\mathrm{MD}=-0.62(-0.88,-0.36)$, $p<0.01$.

Conclusions: Liraglutide effectively improves the lipid profile in patients with NASH.
\end{abstract}

\section{Introduction}

Nonalcoholic steatohepatitis (NASH) is a form of chronic liver disease, which is marked by liver inflammation and results from accumulation of fat in the liver.
It is a progressive form of a group of conditions called nonalcoholic fatty liver disease (NAFLD) [1]. There is a strong association between NASH and metabolic syndromes, particularly obesity and type 2 diabetes [2]. 
NASH can lead to multiple hepatic and cardiovascular complications, which in turn increase the overall mortality rate [3]. Notably, NASH is the commonest cause of chronic liver disease, but no definitive therapies exist for its management or cure.

The first-line treatment for NASH is risk factor modification, which includes management of underlying diabetes and obesity. Weight loss contributes to a reduction of fat and overall inflammation of the liver. Currently, several research studies are being conducted to study the effect of antidiabetic drugs on NASH $[4,5]$. Glucagon-like peptide-1 (GLP-1) analogues, used in type 2 diabetes, have been postulated to play a role in the management of NASH [6]. However, definitive guidelines to direct this therapy are scarce.

Liraglutide is a U.S. Food and Drug Administration (FDA)-approved GLP-1 receptor agonist (GLP-1RA) used in the management of type 2 diabetes mellitus. It acts by glucose-dependent stimulation of insulin secretion, delaying gastric emptying, decreasing appetite, and reducing plasma glucagon concentrations [5]. Delayed gastric emptying and appetite suppression are the main proposed mechanisms responsible for the weight-lowering effects of GLP 1 [5]. In their native forms, GLP-1 analogues have a very short half-life and are metabolized within minutes by dipeptidyl peptidase-4 (DPP-4) enzyme and neutral endopeptidases (NEP) [7]. Therefore, amino acids in liraglutide are substituted and engineered to maximize its peak absorption to $11 \mathrm{~h}$ and absolute bioavailability of $55 \%$, thus allowing for its once-daily subcutaneous dosing.

A study by Gao et al. involving rats showed that liraglutide has anti-oxidative and anti-inflammatory effects in the liver and can consequently reverse hepatic steatosis and insulin resistance [8]. Multiple randomized controlled trials (RCTs) have now been conducted to study the effects and monitor side events of this drug on human subjects.

\section{Aim}

Herein, we perform a meta-analysis to pool the results of similar RCTs available to date in order to report the effect of liraglutide on biochemical markers in patients with biopsy-proven NASH.

\section{Material and methods}

\section{Literature search}

We searched 4 databases: Web of Science, SCOPUS, Cochrane CENTRAL, and PubMed, from inception until October 2020. We followed this search strategy with no restriction on time or languages: liraglutide OR saxenda OR Saxena OR Victoza AND non-alcoholic steatohepatitis. We exported the search results into Endnote X8.0.1
(Build 1044), with the removal of duplicates automatically by computer.

\section{Inclusion and exclusion criteria}

A single investigator reviewed the title and abstracts of all citations identified by our search of the aforementioned 4 databases. The investigator also retrieved and reviewed the full-text versions for the preliminary studies shortlisted initially. The following exclusion and inclusion criteria were applied:

- Inclusion criteria: We included only the RCTs that assessed the efficacy of liraglutide regardless of the dose and the mode of drug administration in patients with NASH. The primary outcomes were change in the biochemical markers: alanine aminotransferase (ALT) (IU/I), aspartate aminotransferase (AST) (IU/l), alkaline phosphatase (ALP) (IU/l), and $\gamma$-glutamyl transferase (GGT) (IU/l). The secondary outcomes included body mass index (BMI) $\left(\mathrm{kg} / \mathrm{m}^{2}\right)$, waist circumference $(\mathrm{cm})$, total cholesterol $(\mathrm{mmol} / \mathrm{l})$, triglyceride $(\mathrm{mmol} / \mathrm{l})$, high-density lipoprotein (HDL) (mmol/l), low-density lipoprotein LDL $(\mathrm{mmol} / \mathrm{l})$, and glycated hemoglobin $\left(\mathrm{HbA}_{1 \mathrm{c}}\right)(\%)$.

- Exclusion criteria: We excluded all non-randomized controlled clinical trials, studies that did not report data or measures for our selected outcomes, and studies with no available full-text forms.

\section{Data extraction}

Two authors (A.M. and W.A.) independently performed the screening steps and searched for the fulltext files of all included studies, which met our selection criteria. A third author (F.I.) solved any deflection. The following data in 2 subgroups was extracted from the selected studies:

1) Baseline and demographic data of patients in each study, including age, sample size, sex, BMI, ALT, AST, GGT, total cholesterol, triglyceride, HDL, LDL, and $\mathrm{HbA}_{1 \mathrm{c}}$.

2) Data for analysis, including outcome values of ALT, AST, AP, GGT, BMI, waist circumference, total cholesterol, triglyceride, $\mathrm{HDL}, \mathrm{LDL}$, and $\mathrm{HbA}_{1 \mathrm{c}}(\%)$.

In addition to the previous 2 categories, we also extracted the data about the 7 domains assessing the risk of bias according to Cochrane's risk of bias [9].

\section{Quality assessment}

We evaluated the quality of this systematic review and meta-analysis using the Grading of Recommendations Assessment, Development, and Evaluation (GRADE) guidelines. We included only the RCTs and excluded the observational evidence. According to the Cochrane risk of bias (ROB) tool for clinical trials, we 


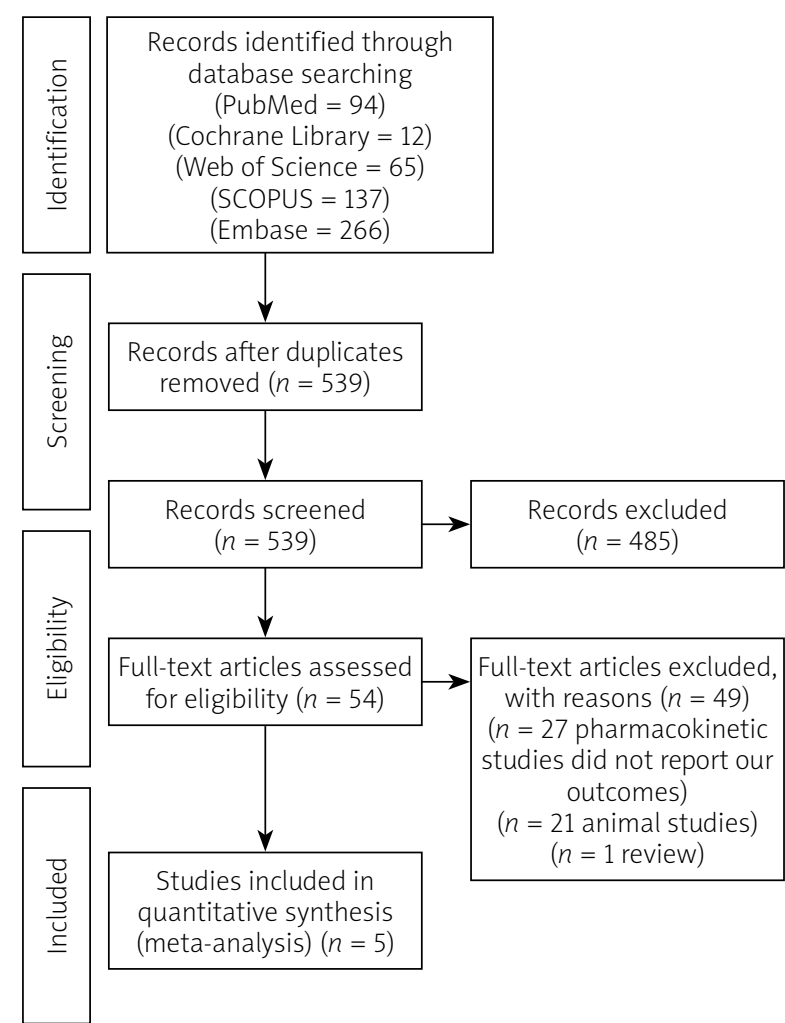

Figure 1. PRISMA flow diagram showing our literature search

performed the risk of bias (ROB) for the included studies. The tool depends on the following domains for assessment of the risk of bias: 1) proper randomization, 2) blinding allocation of the included patients into each group, 3) blinding of patients only (single-blinding), blinding of both personnel and participants (double-blinding), or not blinding at all, 4) attrition bias, $5)$ selection bias (outcomes reported matches with that of the protocol or not), 6) awareness of the outcome assessor (whether blinded or not), and 7) Other bias. The total risk of bias for the studies was also assessed.

\section{Statistical analysis}

We used Review Manager Software (RevMan version 5.4.1) to perform analysis under the inverse variance method. We expressed dichotomous outcomes using percentage and total, while continuous outcomes were described using mean difference (MD) and standard deviation (SD), relative to $95 \%$ confidence interval $(\mathrm{Cl})$. Two main tests indicate inconsistency among studies: the 1 -square test $\left(I^{2}\right)$ and the $p$-value of the $\chi^{2}$ test. The outcomes with $R^{2}>50 \%, p<0.1$ were considered heterogeneous, while outcomes with $l^{2}<50 \%$, $p>0.1$ were considered homogeneous, according to the Cochrane Handbook. Homogenous data were analysed using a fixed-effects model, while heterogene- ous outcomes were analysed using the random-effects model.

This meta-analysis was performed according to the Preferred Reporting Items for Systematic Reviews and Meta-Analyses (PRISMA) [10] and the guidelines reported in the Cochrane Handbook for Systematic Reviews of Interventions [10, 11].

\section{Results}

\section{Selection and characteristics of studies}

The results of the literature search are shown in Figure 1 . The search strategy yielded 45 relevant studies. Five studies met our inclusion criteria. A total of 180 patients were included in these 5 RCTs [12-16]. Of 180 patients, 89 received liraglutide in the treatment group, and 91 patients were assigned to the control group. The mean age of the treatment group was $56.2 \pm 9.18$ years, while that of the control group was $57.7 \pm 9.26$ years. The mean BMI of patients in the liraglutide group was $32.97 \pm 3.8$, while that of the control group was 33.41 \pm 4 . Table I shows a detailed summary of the baseline characteristics of the included studies along with the biochemical markers: ALT, AST, GGT, total cholesterol, triglyceride, $\mathrm{HDL}$, $\mathrm{LDL}$, and $\mathrm{HbA}_{1 \mathrm{c}}$.

\section{Bias assessment}

Cochrane's bias assessment tool yielded an overall low risk of bias. All studies were at low risk of randomization, except Eguchi 2013 [12], which was marked as high risk of bias. For allocation concealment, 2 studies [13, 14] reported adequate allocation concealment; therefore, they were marked to have a low risk of bias. One study [15] did not report enough data about allocation concealment and was thus assigned an unclear risk of bias. Moreover, 2 studies $[12,16]$ reported inadequate allocation concealment. Most of the included studies were blinded. Only one study [12] did not report blindness of the participants and personnel, and it was therefore categorized as an "unclear" risk of bias. Four studies [13-16] were at low risk of blinding of outcome assessment. Eguchi 2013 [12] did not report blindness of outcome assessment, so it was categorized as a "high" risk of bias. The remaining domains of the Cochrane tool were all at low risk of bias. A detailed illustration of the risk of bias of the included trials is summarized in Figure 2.

\section{Outcomes}

ALT (IU/l)

All studies [12-16] reported ALT outcomes. The overall mean difference showed no significant difference between both groups (MD = $2.66(-1.56,6.87), p=0.22)$. Pooled analysis was homogeneous ( $p=0.64 ; 1^{2}=0 \%$ ), as shown in Figure 3. 

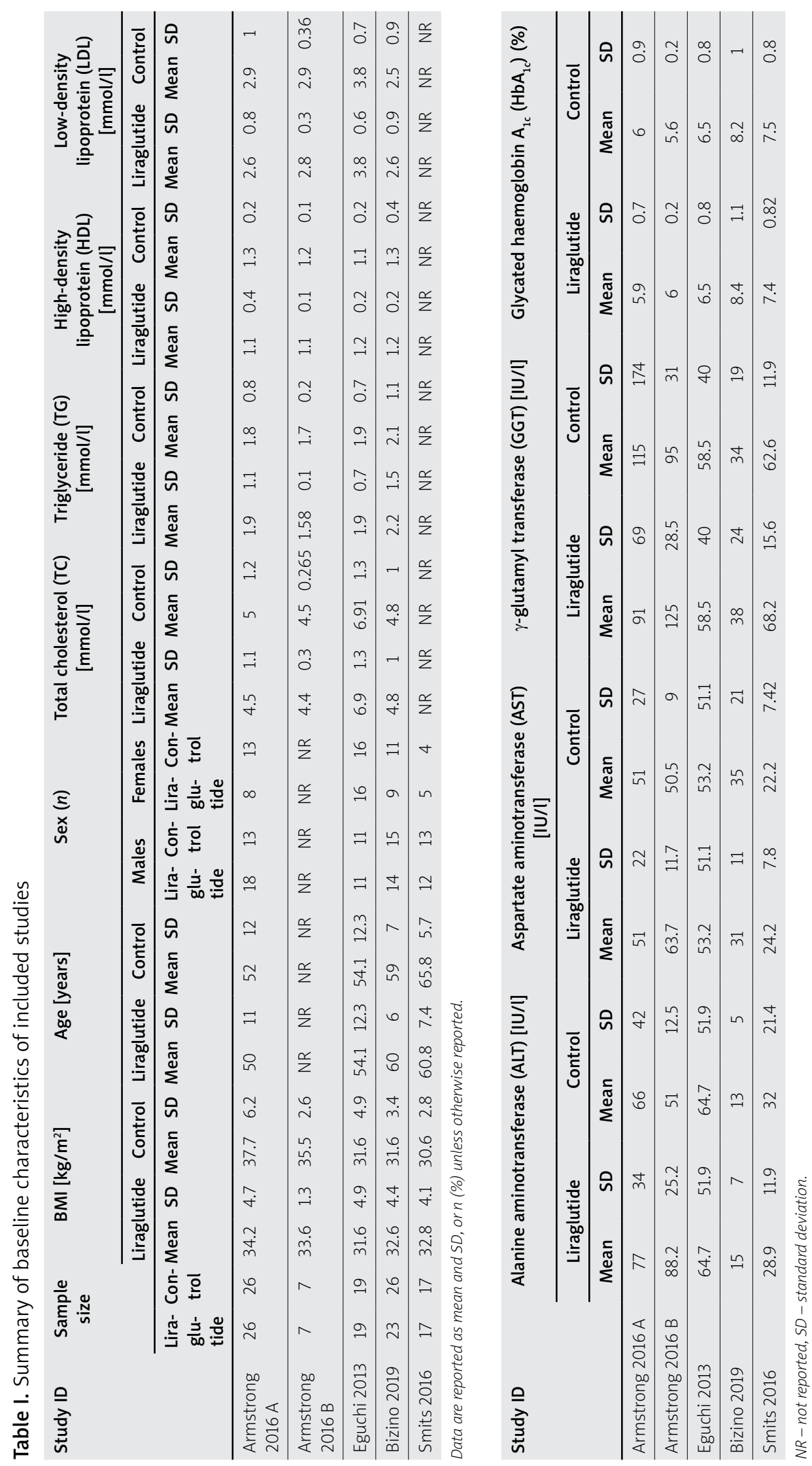


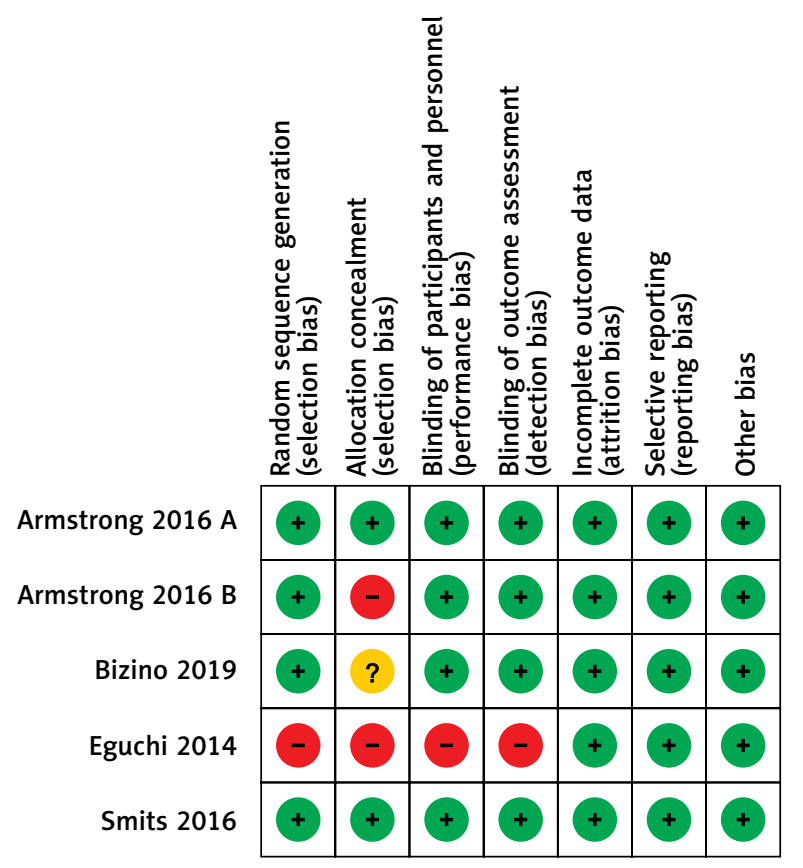

Random sequence generation (selection bias)

Allocation concealment (selection bias)

Blinding of participants and personnel (performance bias)

Blinding of outcome assessment (detection bias)

Incomplete outcome data (attrition bias)

Selective reporting (reporting bias)

Other bias


Sective reporting (reporting bias)

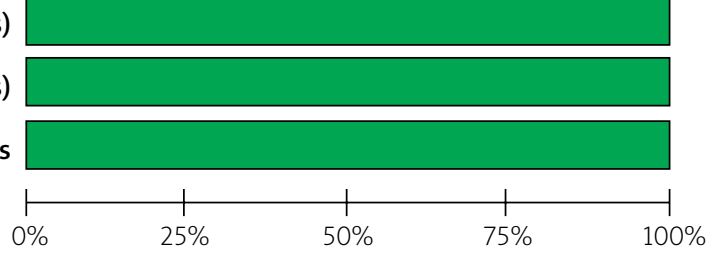

Low risk of bias

Unclear risk of bias

High risk of bias

Figure 2. Risk of bias summary and graph of included studies

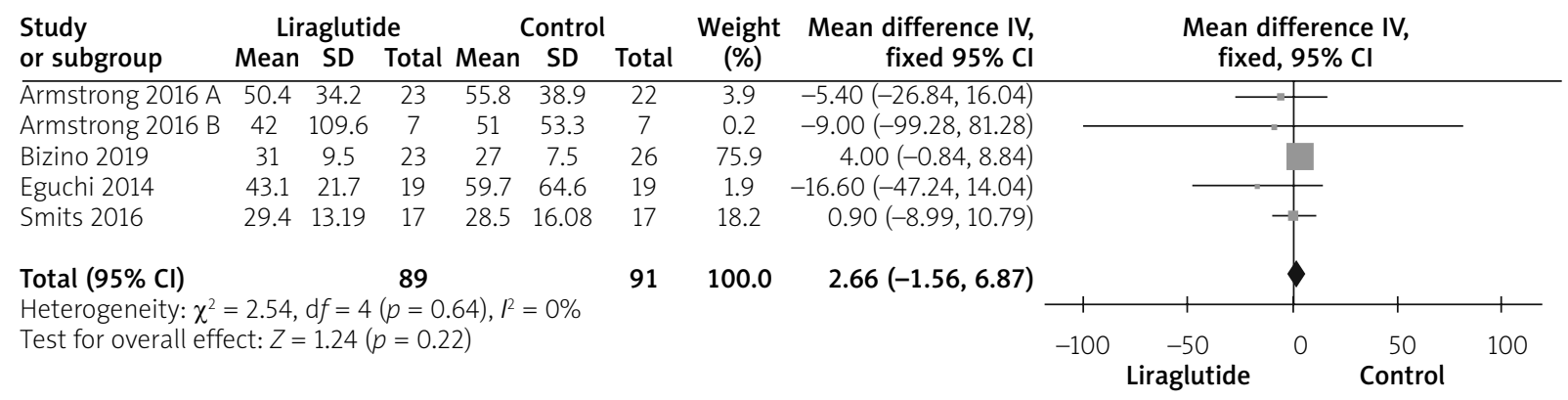

Figure 3. Forest plot for the analysis of alanine aminotransferase (ALT) [IU/I] outcome 


\section{AST (IU/l)}

All studies [12-16] reported AST outcomes. The overall mean difference showed that there was no statistically significant variation between either group $(\mathrm{MD}=-1.99(-5.70,1.72)), p=0.29)$. Data were homogeneous $\left(p=0.13 ; r^{2}=43 \%\right)$, as shown in Figure 4 .

\section{GGT $(I U / l)$}

The GGT was reported by all studies [12-16]. The combined mean difference did not reveal a statistically significant difference between both groups (MD $=5.02$ $(-0.86,10.90), p=0.09)$. Pooled analysis was homogeneous $\left(p=0.12 ; 1^{2}=45 \%\right)$, as shown in Figure 5 .

\section{$A L P(I U / l)$}

Four studies reported alkaline phosphatase outcomes [13-16]. The overall analysis did not show any statistically significant variation between both groups $(\mathrm{MD}=-5.16(-11.90,1.59), p=0.13)$. Data were homogeneous $\left(p=0.28 ; l^{2}=22 \%\right)$, as shown in Figure 6 .

\section{Total cholesterol (mmol/l)}

Four studies [12, 14-16] reported the total cholesterol outcome. The overall mean difference showed no statistically significant difference between both groups $(\mathrm{MD}=-0.31(-0.65,0.03), p=0.07)$. Pooled analysis was homogeneous ( $\left.p=0.93 ; 1^{2}=0 \%\right)$, as shown in Figure 7 .

\section{Triglyceride (mmol/l)}

Four studies [12, 14-16] reported serum triglyceride levels. Pooled analysis showed no statistically significant difference between either group $(M D=-0.14$ $(-0.53,0.25), p=0.48)$. Pooled analysis was homogeneous $\left(p=0.83 ; 1^{2}=0 \%\right)$, as shown in Figure 8 .

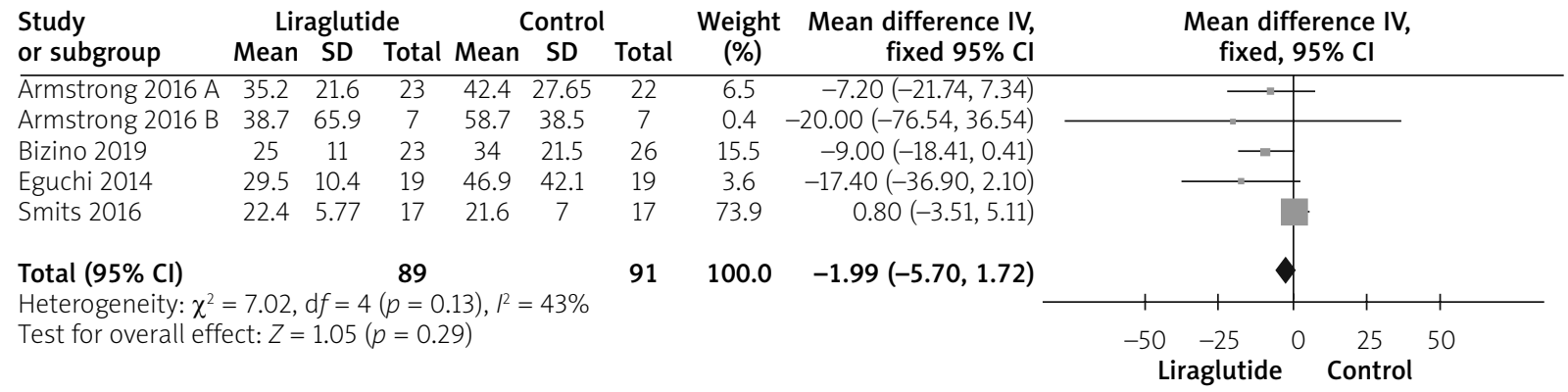

Figure 4. Forest plot for the analysis of aspartate aminotransferase (AST) [IU/I] outcome

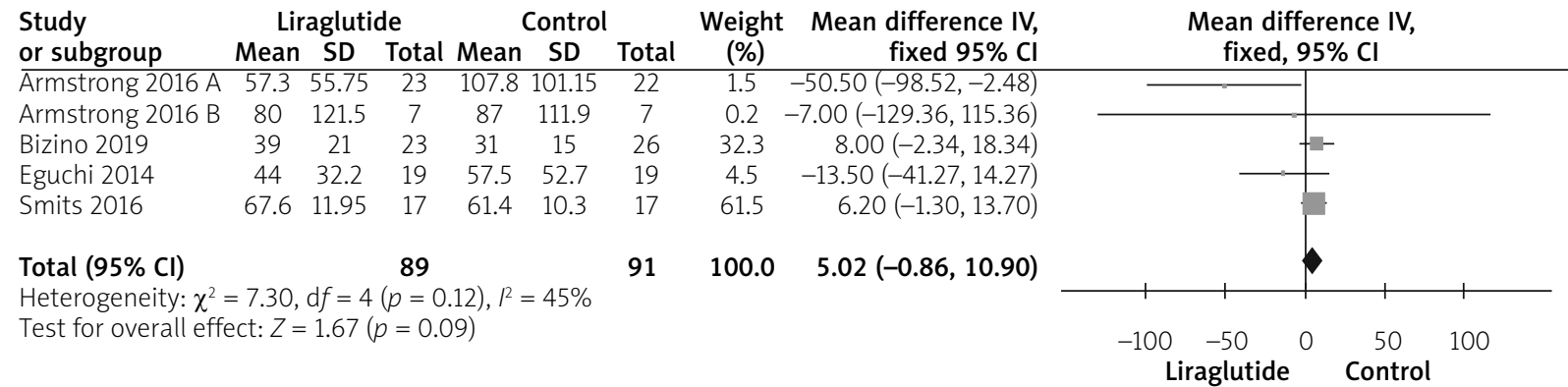

Figure 5. Forest plot for the analysis of $\gamma$-glutamyl transferase (GGT) [IU/I] outcome

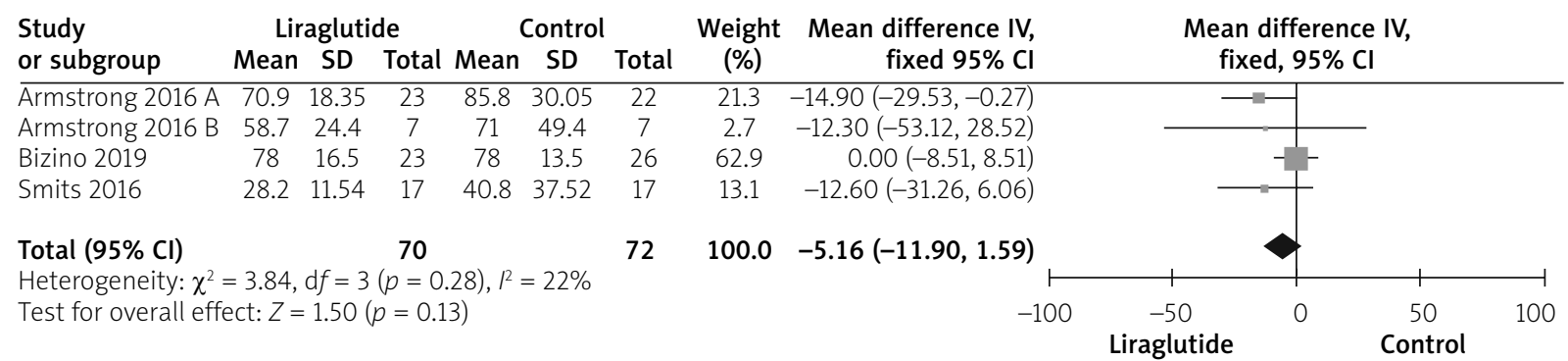

Figure 6. Forest plot for the analysis of alkaline phosphatase (ALP) [IU/I] outcome 


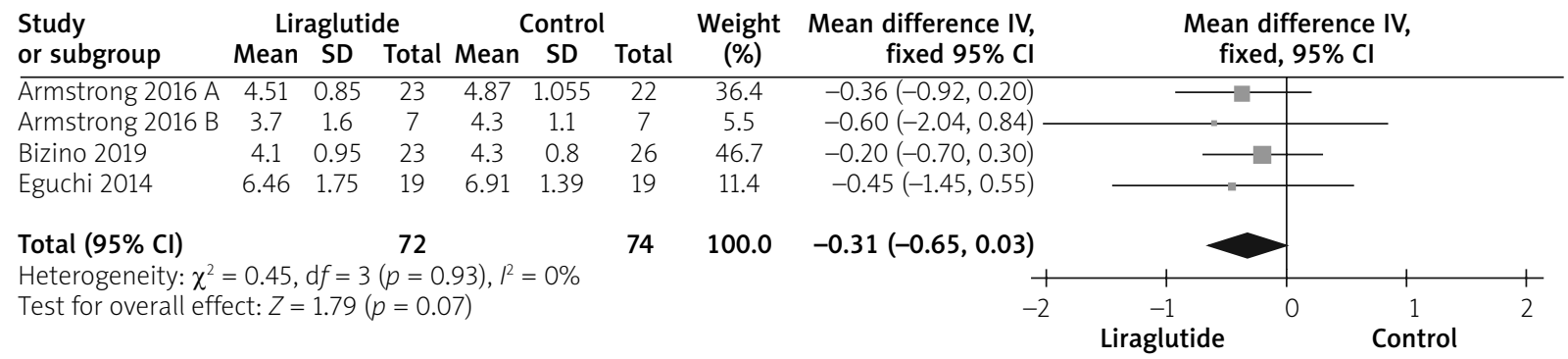

Figure 7. Forest plot for the analysis of total cholesterol [mmol/l] outcome

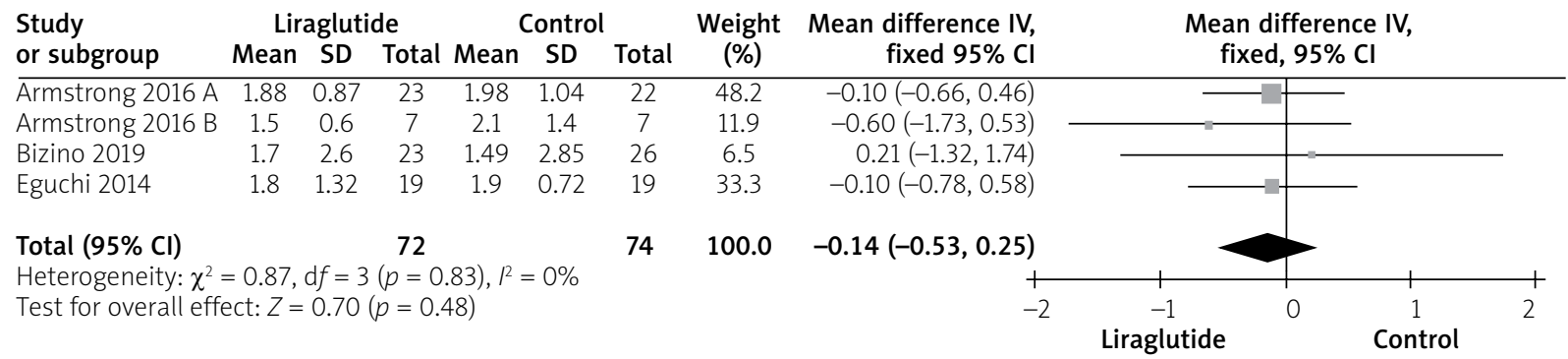

Figure 8. Forest plot for the analysis of triglyceride [mmol/l] outcome

\section{HDL (mmol/l)}

Four studies [12, 14-16] reported HDL outcomes. The overall mean difference favoured the liraglutide group over the control group $(M D=0.10(0.02,0.18)$, $p=0.02)$. Data were homogeneous $\left(p=0.35 ; 1^{2}=8 \%\right)$, as shown in Figure 9.

\section{LDL (mmol/l)}

Four studies [12, 14-16] reported the LDL outcomes. The liraglutide group showed statistically significant reduction in LDL (MD $=-0.29(-0.56,-0.02)), p=0.04)$. Pooled analysis was homogeneous ( $p=0.49 ; l^{2}=0 \%$ ), as shown in Figure 10.



Figure 9. Forest plot for the analysis of high-density lipoprotein (HDL) [mmol/l] outcome

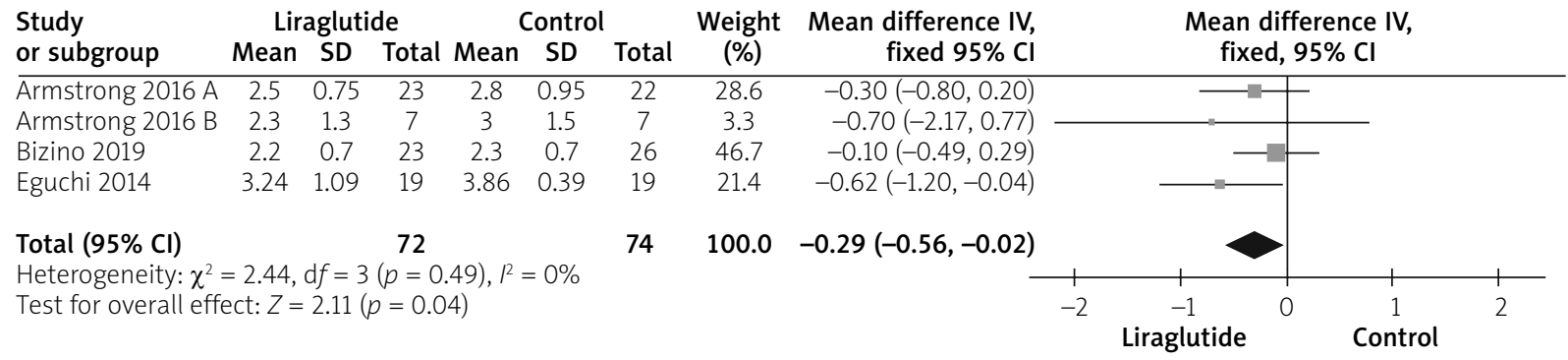

Figure 10. Forest plot for the analysis of low-density lipoprotein $L D L[\mathrm{mmol} / \mathrm{l}]$ outcome 
$H b A_{1 c}(\%)$

The $\mathrm{HbA}_{1 \mathrm{c}}$ were reported by all studies $[12,14-16]$. The pooled analysis favoured the liraglutide group over the control group significantly $(M D=-0.62(-0.88$, $-0.36), p<0.01)$. Pooled analysis was homogeneous $\left(p=0.22 ; 1^{2}=30 \%\right)$, as shown in Figure 11 .

\section{$B M I\left(\mathrm{~kg} / \mathrm{m}^{2}\right)$}

BMIs were reported by all studies [12-16]. The overall mean difference did not reveal any statistically significant difference between both groups ( $M D=-1.50$ $(-3.95,0.95), p=0.23)$, as shown in Figure 12. Pooled analysis was heterogeneous ( $\left.p=0.08 ; 1^{2}=79 \%\right)$, as shown in Figure $12 \mathrm{~A}$. We solved the heterogeneity by the exclusion of Armstrong $2016 \mathrm{~A}(2)(p=0.28) ; 1^{2}=$ $22 \%$. However, the pooled analysis after exclusion still showed no statistically significant difference between either group $(M D=-0.26(-1.68,1.16) ; p=0.72)$. Figure $12 \mathrm{~B}$ illustrates the analysis after the exclusion of the study.

\section{Waist circumference $(\mathrm{cm})$}

Three studies reported the waist circumference measurements [14-16]. The combined mean difference did not reveal a statistically significant difference between both groups ( $M D=-6.19(-14.70,2.32)$; $p=0.15)$ as shown in Figure 13. Pooled data were heterogeneous $\left(p=0.02 ; r^{2}=75 \%\right.$ ), as shown in Figure $13 \mathrm{~A}$. We solved the heterogeneity by exclusion of Bizini $2019[15]\left(p=0.94 ; 1^{2}=0 \%\right)$. The pooled analysis after the exclusion favoured the liraglutide group over the control group (MD $=-10.96(-16.79,-5.14) p=0.02)$. Figure $13 \mathrm{~B}$ illustrates the analysis after the exclusion of the study.

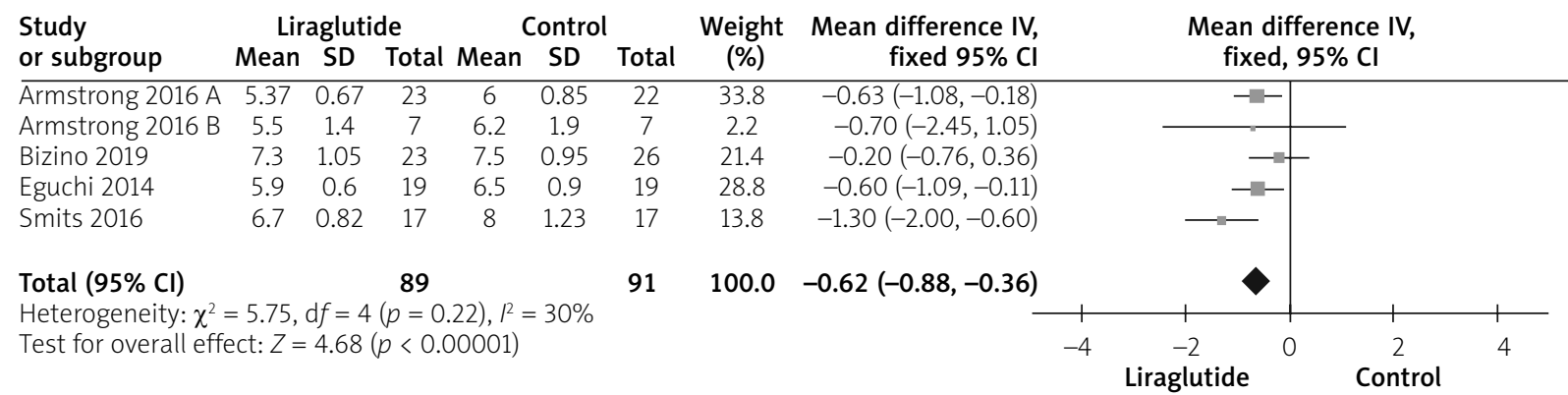

Figure 11. Forest plot for the analysis of $\mathrm{HbA}_{1 \mathrm{c}}$ (\%) outcome

\section{A}

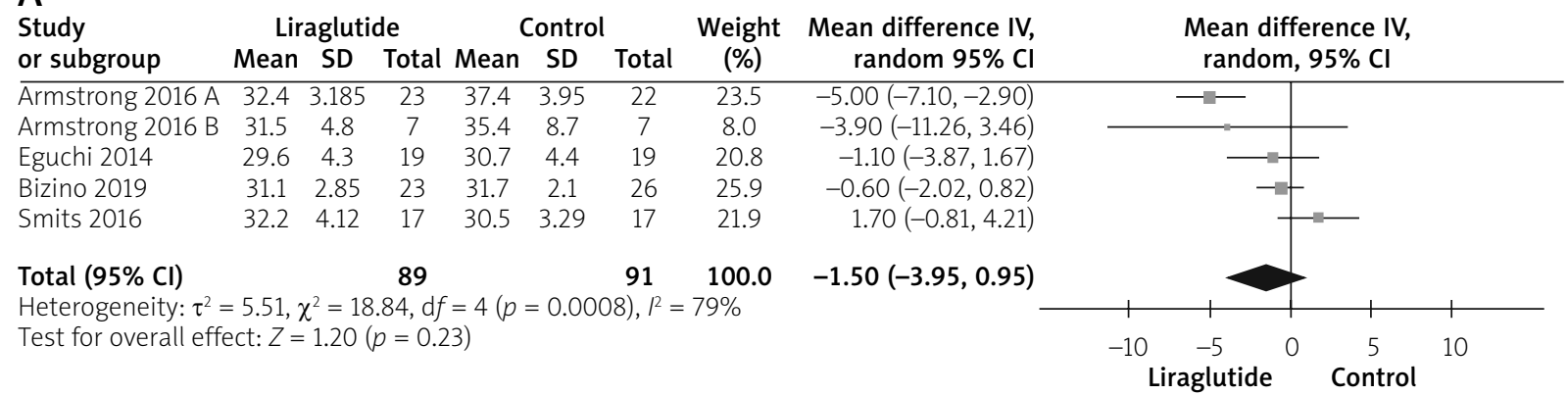

B

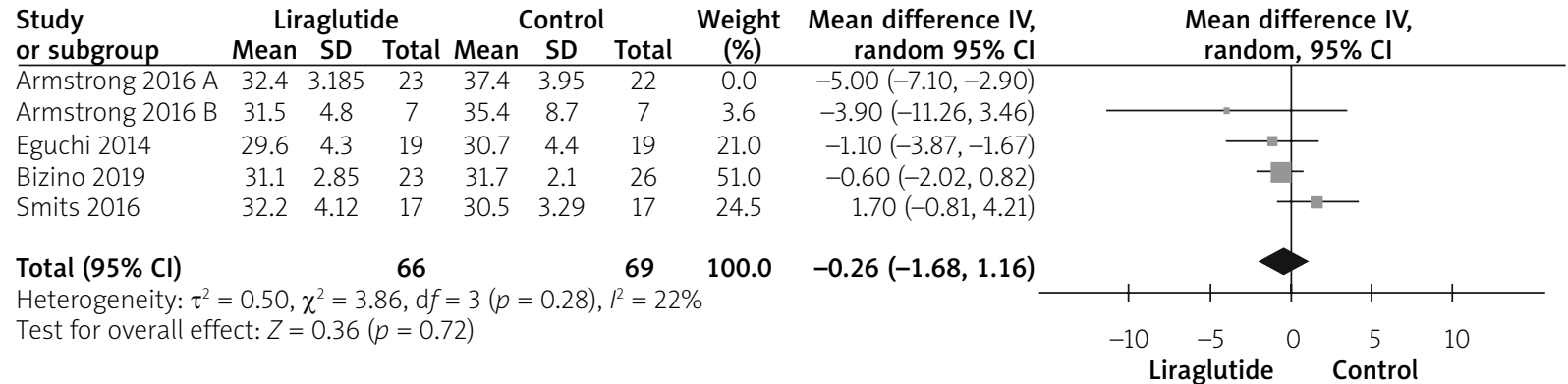

Figure 12. Forest plot for the analysis of body mass index (BMI) $\left[\mathrm{kg} / \mathrm{m}^{2}\right]$ outcome: $\mathbf{A}$ - before leave-one-out, B - after leave-one-out 


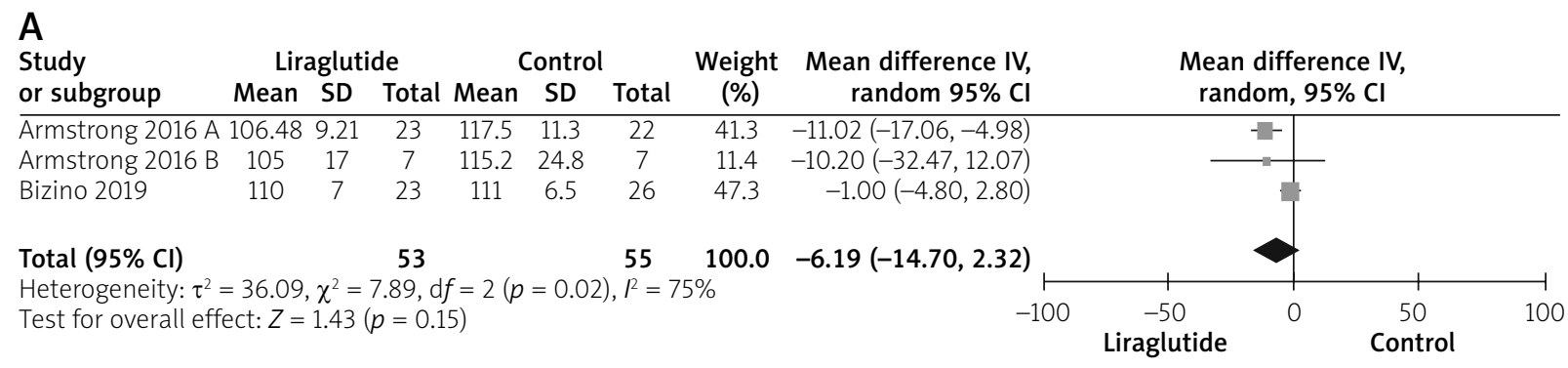

\section{$B$}

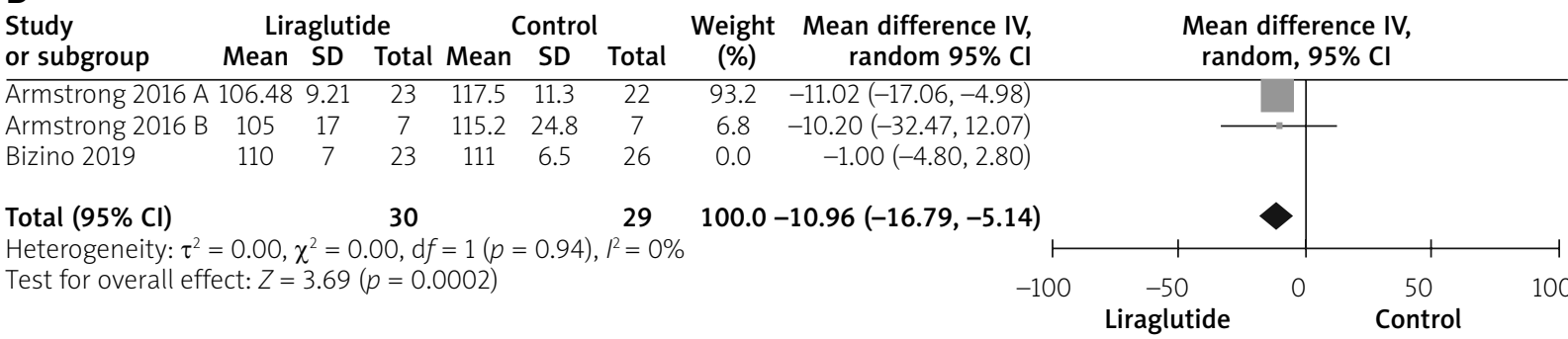

Figure 13. Forest plot for the analysis of waist circumference $[\mathrm{cm}]$ outcome: $\mathbf{A}$ - before leave-one-out,

B - after leave-one-out

\section{Discussion}

Our meta-analysis of the 5 RCTs shows that liraglutide is effective in improving the lipid profile in patients with NASH [12-16]. This is shown by the statistically significant decrease in LDL levels $(M D=-0.29(-0.56$, $-0.02) ; p=0.04)$ and improvements in HDL levels $(M D=0.10(0.02,0.18) ; p=0.02)$ in the blood. Liraglutide also showed a statistically significant reduction in $\mathrm{HbA}_{1 \mathrm{c}}$ levels in patients, thus expressing its known antidiabetic effects. However, compared to placebo, liraglutide was not statistically significant in reducing liver enzymes, ALT, AST, GGT, or ALP. The analysis also showed a decrease in levels of total cholesterol and total triglycerides; however, it was not found to be statistically significant.

Our results are similar to studies published in the literature. Previously published trials have shown that liraglutide is not effective in improving levels of ALT, AST, GGT, and AP [12, 14-16]. Except for Armstrong et al. [16], who reported improvements in GGT and ALT levels. Total cholesterol and total triglyceride levels were not statistically significantly different between all included studies' intervention and control arms [12-16]. Regarding $\mathrm{HbA}_{1 \mathrm{c}}$ levels, 2 studies reported a non-significant difference $[13,15]$, while 3 studies showed that liraglutide reduces $\mathrm{HbA}_{1 c}$ levels significantly $[12,14,16]$.

This study is an updated meta-analysis specifically performed to investigate the role of liraglutide in patients with NASH. However, liraglutide has been used in the management of many other systemic conditions and diseases. A meta-analysis by Armstrong et al. con- cluded that liraglutide $1.8 \mathrm{mg}$ is safe, well-tolerated, and improves liver enzymes in patients with type 2 diabetes and elevated liver enzymes [17]. Another meta-analysis by Zhang et al. found liraglutide to be an effective and safe treatment for weight loss in obese, non-diabetic individuals [18]. Our results demonstrate that liraglutide reduces $\mathrm{HbA}_{1 c}$ significantly compared to placebo. However, the meta-analysis by Morieri et al. revealed that liraglutide has lower efficacy in lowering $\mathrm{HbA}_{1 \mathrm{c}}$ than other glucagon-like peptide-1 receptor agonists, such as dulaglutide [19].

Liraglutide has also been shown to improve cardiac function in patients with heart failure [20]. Regarding safety endpoints, it decreases the risk of major adverse cardiovascular events and all-cause mortality [21]. Liraglutide is not related to the risk of acute pancreatitis or cancer [22]. Davidson et al. found that mild renal impairment had no effect on the efficacy and safety of liraglutide in their meta-analysis [23].

The main strength of this study is the inclusion of randomized clinical trials only and the exclusion of observational evidence. It is well-known that RCTs exhibit the highest level of evidence, according to the Cochrane Handbook. Another point is that all our analysis results were homogeneous, which demarcates the consistency of data and supports the interpretation of the analysis results. According to Cochrane's risk of bias tool, we also assessed the risk of bias among the included studies and found it to be low. However, although we included all previously published clinical trials, the small number of included studies [5] remains a significant 
limitation. Another limitation is the small sample size (180 patients), which reduces our findings' generalizability. Therefore, more clinical trials are still needed to investigate the efficacy of the drug. Additionally, the included studies did not report many data about liraglutide side effects, which further necessitates clinical trials providing a detailed safety profile.

\section{Conclusions}

Liraglutide appears to effectively improve lipid profile (HDL and LDL) in patients with NASH. However, its effect is not remarkable in reducing liver enzymes. While there is currently a paucity of data on liraglutide efficacy, further research may prove it to be a potential therapy for the prevention of NASH progression and/or disease reversal.

\section{Acknowledgments}

The preliminary form of these data was presented as an abstract at the American Association for the Study of Liver Diseases (AASLD) - The Liver Meeting, 12-15 November 2021, in Anaheim, CA, USA.

\section{Conflict of interest}

The authors declare no conflict of interest.

\section{References}

1. Benedict M, Zhang X. Non-alcoholic fatty liver disease: an expanded review World J Hepatol 2017; 9: 715-32.

2. Godoy-Matos AF, Silva Júnior WS, Valerio CM. NAFLD as a continuum: from obesity to metabolic syndrome and diabetes. Diabetol Metab Syndr 2020; 12: 60.

3. Armstrong MJ, Adams LA, Canbay A, et al. Extrahepatic complications of nonalcoholic fatty liver disease Hepatology 2014; 59: 1174-97.

4. Sberna AL, Bouillet B, Rouland A, et al. European Association for the Study of the Liver (EASL), European Association for the Study of Diabetes (EASD) and European Association for the Study of Obesity (EASO) clinical practice recommendations for the management of non-alcoholic fatty liver disease: evaluation of their application in people with type 2 diabetes. Diabet Med 2018; 35: 368-75.

5. Byrne CD, Targher G. EASL-EASD-EASO Clinical Practice Guidelines for the management of non-alcoholic fatty liver disease: is universal screening appropriate? Diabetologia 2016; 59: 1141-4.

6. Armstrong MJ, Gaunt P, Aithal GP, et al. OC-016 Liraglutide is effective in the histological clearance of non-alcoholic steatohepatitis in a multicentre, double-blinded, randomised, placebo-controlled phase II trial. Gut 2015; 64: A9.

7. Ampuero J, Sánchez-Torrijos Y, Aguilera V, et al. New therapeutic perspectives in non-alcoholic steatohepatitis. Gastroenterol Hepatol 2018; 41: 128-42.

8. Chaston TB, Dixon JB. Factors associated with percent change in visceral versus subcutaneous abdominal fat during weight loss: findings from a systematic review Int J Obes 2008; 32 : 619-28.

9. Munder T, Barth J. Cochrane's risk of bias tool in the context of psychotherapy outcome research Psychother Res. 2018; 28 : 347-55.

10. Moher D, Liberati A, Tetzlaff J, et al. Preferred reporting items for systematic reviews and meta-analyses: the PRISMA statement PLoS Med 2009; 6: se1000097.

11. Higgins JPT, Chandler J, Cumpston M, et al. Cochrane Handbook for Systematic Reviews of Interventions version 6.2 (updated February 2021). Cochrane, 2021. City: John Wiley \& Sons, 2019.; 2021.

12. Eguchi Y, Kitajima Y, Hyogo H, et al. Pilot study of liraglutide effects in non-alcoholic steatohepatitis and non-alcoholic fatty liver disease with glucose intolerance in Japanese patients (LEAN-J) Hepatol Res 2015; 45: 269-78.

13. Armstrong MJ, Hull D, Guo K, et al. Glucagon-like peptide 1 decreases lipotoxicity in non-alcoholic steatohepatitis. J Hepatol 2016; 64: 399-408.

14. Smits MM, Tonneijck L, Muskiet MH, et al. Twelve week liraglutide or sitagliptin does not affect hepatic fat in type 2 diabetes: a randomised placebo-controlled trial. Diabetologia 2016; 59: 2588-93.

15. Bizino MB, Jazet IM, de Heer P, et al. Placebo-controlled randomised trial with liraglutide on magnetic resonance endpoints in individuals with type 2 diabetes: a pre-specified secondary study on ectopic fat accumulation Diabetologia 2020; 63: 65-74.

16. Armstrong MJ, Gaunt P, Aithal GP, et al. Liraglutide safety and efficacy in patients with non-alcoholic steatohepatitis (LEAN): a multicentre, double-blind, randomised, placebo-controlled phase 2 study. Lancet 2016; 387: 679-90.

17. Armstrong MJ, Houlihan DD, Rowe IA, et al. Safety and efficacy of liraglutide in patients with type 2 diabetes and elevated liver enzymes: individual patient data meta-analysis of the LEAD program. Aliment Pharmacol Ther 2013; 37: 234-42.

18. Zhang P, Liu Y, Ren Y, et al. The efficacy and safety of liraglutide in the obese, non-diabetic individuals: a systematic review and meta-analysis. Afr Health Sci 2019; 19: 2591-9.

19. Morieri ML, Rigato M, Frison V, et al. Effectiveness of dulaglutide vs liraglutide and exenatide once-weekly. A real-world study and meta-analysis of observational studies. Metabolism 2020; 106: 154190.

20. Zhang Y, Chen Q, Huang G, et al. The influence of liraglutide for heart failure: a meta-analysis of randomized controlled trials. Heart Surg Forum 2019; 22: E438-44.

21. Duan CM, Wan TF, Wang Y, et al. Cardiovascular outcomes of liraglutide in patients with type 2 diabetes: a systematic review and meta-analysis. Medicine 2019; 98: e17860.

22. Alves C, Batel-Marques F, Macedo AF. A meta-analysis of serious adverse events reported with exenatide and liraglutide: acute pancreatitis and cancer. Diabetes Res Clin Pract 2012; 98: 271-84.

23. Davidson JA, Brett J, Falahati A, et al. Mild renal impairment and the efficacy and safety of liraglutide Endocr Pract 2011; 17: 345-55.

Received: 8.12.2021

Accepted: 16.12 .2021 